\title{
Three-Dimensional Characterization of Carotid Plaque Structure and Deformation with Non-Invasive Ultrasound Imaging
}

\author{
${ }^{*} \mathrm{~K}_{\text {Dionisio }}{ }^{1}{ }^{*} \mathrm{R}$ Chan ${ }^{1,2}$, LW Campbell ${ }^{1}, \mathrm{~J} \mathrm{Connelly}^{1}, \mathrm{WC} \mathrm{Karl}^{3}$, RS Lees ${ }^{1}$ \\ ${ }^{1}$ Harvard-MIT Division of Health Sciences and Technology, Cambridge, MA, USA \\ ${ }^{2}$ Department of Radiology, Massachusetts General Hospital, Boston, MA, USA \\ ${ }^{3}$ Department of Electrical and Computer Engineering, Boston University, Boston, MA, USA
}

\begin{abstract}
We have shown that by using a directionally sensitive, edge preserving filter on B-mode U/S images of ex-vivo carotid vessels we can obtain $3 D$ edge fields of the vessel boundaries that are more distinct than edges from conventional gradient calculation. With these enhanced edge fields we can then extract the $3 D$ vessel boundaries. BlandAltman plots show that our method for edge field enhancement and boundary extraction yields more reproducible IMT measurements than the gold standard of manual point selection. Lastly, we demonstrate a robust estimator of velocity and strain that exhibits enhanced performance over conventional methods.
\end{abstract}

\section{Introduction}

Atherosclerosis causes heterogeneous remodeling of arterial structure and composition. As a consequence, the interrogation of arterial morphology and biomechanics plays an important role in monitoring the natural history of atherogenesis and in quantifying drug efficacy based on image-derived endpoints. Non-invasive peripheral vascular ultrasound in the carotid provides a cost-effective means of estimating atherosclerotic disease burden and has been used extensively for quantifying mean intimamedia thickness (IMT) and vessel diameter distension in two-dimensional longitudinal scans of the distal common carotid artery [1]. These measurements are however constrained to a relatively small section of the carotid lying within the imaging plane and therefore are scan plane dependent. With the advent of three-dimensional (3D) freehand ultrasound imaging, more comprehensive evaluation of disease-induced alterations in vessel morphology and biomechanics is now possible.

Accurate and reproducible characterization of carotid structure and mechanical properties from these 3D ultrasonic data is essential since it would not only allow for better plaque quantification than routine clinical methods, but would also provide a cost-effective alternative to vascular magnetic resonance imaging for patient-specific computational modeling of atherogenesis [2]. The analysis of carotid morphology and dynamic deformation from peripheral vascular ultrasound is challenging however due to the presence of speckle noise from the coherent imaging process, electronic noise from imaging instrumentation, and other imaging artifacts. Manual tracing remains the gold-standard for image analysis in this view despite the wide recognition that it is time consuming and subjective, leading to results which vary depending on viewer experience and perception.

Development of methods to improve the measurement of carotid morphology and deformation in noisy short-axis imaging views is clearly necessary to realize fully the potential of 3D carotid ultrasound imaging. In this work, we present a processing scheme that addresses the need for robust ultrasound-based characterization of carotid structure and deformation in 3D.

\section{Methods}

\section{Data acquisition}

Fresh carotid specimens were harvested from autopsy starting at the distal common carotid and extending through the bulb, and proximal internal and external carotid branches. Specimens were immersed in phosphate buffered saline (PBS) and stored in the refrigerator at $4^{\circ} \mathrm{C}$ for imaging within 24 hours of harvesting. Pressure tubing connectors were inserted into the common, internal, and external carotid arteries and secured with suture. Carotid side-branches were tied off with suture to prevent leakage during pressurization.

Following preparation, the sample was mounted within a specimen holder under slight tension and immersed into a shallow saline bath for imaging. A saline bag and a digital manometer (MP2000.001, APT Instruments) were attached to the pressure tubing connector at the distal common carotid and the pressure tubing connectors at the internal and external carotid arteries were sealed with press- 
in plugs. With this arrangement, the hydrostatic pressure within the carotid could be controlled systematically by changing the height of the saline bag relative to the specimen. The corresponding pressure was simultaneously recorded by the digital manometer. Ultrasound (U/S) scanning of the carotid specimen in short-axis was performed with a broadband 5-12 MHz peripheral vascular transducer and the resulting B-mode images were digitized from a Philips HDI 5000 Sono CT machine (ATL-Philips Ltd., Bothell, WA). Transducer position and orientation were monitored using the Flock of Birds (Ascension Technologies Inc., VT) so that pixels could be mapped to 3D spatial coordinates within the BIRD transmitter frame-ofreference.

Stradx V.7.1 (Cambridge University, UK) was used for acquisition of imaging data and corresponding position measurements. A custom-built transducer mount, which allowed for smooth manual translation of the transducer at a fixed distance over the specimen, was used as an aid to freehand scanning. Finally, within Stradx, all data sets were voxelized onto a rectilinear grid with a voxel size of $0.079 \mathrm{~mm}$ on each side.

\section{Vessel morphology estimation}

Our approach to carotid segmentation is based on a nonlinear, variational energy minimization first proposed by Shah [3]. We have modified this functional for incorporation of direction-sensitive smoothing and edge preservation in 3D based on user-specified prior information about arterial shape. This energy minimization approach allows for the use of global voxel information in volume restoration and edge field estimation, as opposed to linear filters which smooth noisy data and compute gradients based only on local voxel characteristics.

The process begins with user-specified initial surfaces for the inner and outer boundaries, roughly indicating the orientation of the vessel boundary. The volumetric data are then resampled with trilinear interpolation along the initial surface normals so that in the resampled space, the "desired" edge orientation corresponds to a horizontally-aligned plane, regardless of the prior contour shape in the original space. We then perform anisotropic smoothing and edge enhancement in this space, using a new directionally-sensitive energy functional $E_{\mathrm{DS}}$ shown in Eq.1.

$$
\begin{aligned}
E_{\mathrm{DS}}(f, s)= & \int_{V} \underbrace{(1-s)^{2}\{D(f)\}}_{\text {Smoothness constraint }} \\
& +\underbrace{\beta\|f-g\|_{1}}_{\text {Data fidelity }}+\underbrace{\frac{\rho}{2}\|\nabla s\|_{2}^{2}+\frac{s^{2}}{2 \rho}}_{\text {Edge penalty }} d V \\
D(f)= & \alpha_{x}\left\|\frac{\partial f}{\partial x}\right\|_{1}+\alpha_{y}\left\|\frac{\partial f}{\partial y}\right\|_{1}+\alpha_{z}\left\|\frac{\partial f}{\partial z}\right\|_{1}
\end{aligned}
$$

In Eq.(1), the notation, $\|\cdot\|_{1}$, represents an $l_{1}$ norm, and $\|\cdot\|_{2}$ represents an $l_{2}$ norm. The notation $V$ represents the volume domain of interest, $g$ is the resampled ultrasound volume, $f$ is the anisotropically-smoothed field which represents a piecewise-constant approximation to the noisy observations, and $s$ is the estimated volume edge field with values ranging between 0 and 1 .

The smoothness constraint term penalizes large gradients in $f$ except where edges exist $(s \sim 1)$. This edge dependency results in anisotropic smoothing that is greatest far away from structure boundaries. The separate smoothness weighting parameters $\alpha_{x}, \alpha_{y}$, and $\alpha_{z}$ allow for direction-sensitive edge preservation. To enhance horizontally aligned surfaces in the resampled volume, $\alpha_{x}$ and $\alpha_{z}$ are specified to be much larger than $\alpha_{y}$. The data fidelity component controls the degree to which the smoothed field $f$ resembles the data, $g$. The edge penalty term controls the edge width and prevents the minimization process from placing edges everywhere in the edge field (the trivial solution $s=1, \forall(x, y) \in R)$. The real positive scalars $\alpha_{x}, \alpha_{y}, \alpha_{z}, \beta$, and $\rho$ adjust the relative weighting between these competing terms. In the limit as $\rho \rightarrow 0$, shocks or intensity discontinuities develop at boundaries, allowing for sharp demarcation of volume regions.

Numerical solution of Eq.(1) is performed by iterative minimization of the discretized energy functional with a coordinate descent approach. Iteration continues until convergence to a predefined tolerance level is achieved. Additional details of this process can be found in [4]. After the final estimates for the restored (noise suppressed) volume $f$ and the volume edge field $s$ have been obtained, both fields are mapped back into the original volume domain for visualization and vessel boundary extraction with 3D deformable surfaces.

The sparse volume edges from 3D direction-sensitive smoothing and edge enhancement are used together with the initial surface locations to evolve deformable surfaces at the inner and outer vessel borders. These deformable surfaces, $\mathbf{x}\left(t_{1}, t_{2}\right)=\left[x\left(t_{1}, t_{2}\right), y\left(t_{1}, t_{2}\right), z\left(t_{1}, t_{2}\right)\right],\left(t_{1}, t_{2} \in\right.$ $[0,1])$ move over the domain of the ultrasound volume to minimize the energy functional:

$$
\begin{aligned}
E_{\text {deform }}(\mathbf{x})= & \int_{t_{1}} \int_{t_{2}} \frac{1}{2} a\left\{\left\|\frac{\partial \mathbf{x}}{\partial t_{1}}\right\|_{2}^{2}+\left\|\frac{\partial \mathbf{x}}{\partial t_{2}}\right\|_{2}^{2}\right\} \\
& +\frac{1}{2} b\left\{\left\|\frac{\partial^{2} \mathbf{x}}{\partial t_{1}^{2}}\right\|_{2}^{2}+\left\|\frac{\partial^{2} \mathbf{x}}{\partial t_{2}^{2}}\right\|_{2}^{2}+\left\|\frac{\partial^{2} \mathbf{x}}{\partial t_{1} \partial t_{2}}\right\|_{2}^{2}\right\}- \\
& G_{\sigma}(x, y, z) * s(x, y, z) d t_{1} d t_{2}
\end{aligned}
$$

$G_{\sigma}(x, y, z)$ is a 3D Gaussian with a standard deviation $\sigma$ which is convolved with the estimated $3 \mathrm{D}$ edge field $s$ to control the capture range of the deformable surface. The edge field acts to pull points on the deformable surface 
towards strong boundary gradients, while the deformable surface simultaneously adapts its shape to minimize the energy associated with surface stretching and bending. Following extraction of inner and outer carotid wall surfaces, the carotid wall thickness is computed for every shortaxis cross-section by calculating the Euclidean distance between the inner and outer borders at each circumferential location around the vessel wall.

\section{Vessel motion and strain estimation}

Ultrasound-based estimation of tissue motion within a vessel cross-section is performed by estimating the velocity, $(u, v)$, that maximizes the correlation coefficient, $\rho$, between image blocks of pre-defined size in an initial reference frame and corresponding blocks to which tissue has displaced in a subsequent search frame. This velocity estimator is expressed mathematically in Eq.(4) for an image block centered at $(x, y)$ within the reference image. The desired velocity estimates, $\hat{u}$ and $\hat{v}$, in the $x$ and $y$ directions respectively, will maximize the correlation coefficient function $\rho_{x, y}(u, v)$

$$
\begin{gathered}
{\left[\begin{array}{ll}
\hat{u} & \hat{v}
\end{array}\right]=\arg \max \rho_{x, y}(u, v)} \\
{\left[\begin{array}{cc}
u & v
\end{array}\right]}
\end{gathered}
$$

This approach to velocity and strain estimation is not robust however to imaging noise typical of peripheral vascular ultrasound. Therefore, we describe here the use of a velocity and strain estimator recently developed by Chan et al for tissue elastography with optical coherence tomography [5]. We estimate the velocity field that minimizes the following variational energy functional

$$
E(V(x, y))=\theta E_{D}(V(x, y))+\omega E_{S}(V(x, y))+\psi E_{I}(V(x, y))
$$

This energy depends on the desired velocity distribution $V(x, y)=\left[\begin{array}{ll}u(x, y) & v(x, y)\end{array}\right]$ and consists of three terms which reflect data fidelity, $E_{D}(V(x, y))$, strain field smoothness, $E_{S}(V(x, y))$, and tissue incompressibility, $E_{I}(V(x, y))$, as expressed by the following functions:

$$
\begin{aligned}
& E_{D}(V(x, y))=-\iint \rho_{x, y}(V(x, y)) d x d y \\
& E_{S}(V(x, y))=\iint\left\|\nabla^{2} V(x, y)\right\|^{2} d x d y \\
& E_{I}(V(x, y))=\iint\|\nabla \cdot V(x, y)\|^{2} d x d y
\end{aligned}
$$

Minimizing the data fidelity term is identical to maximizing the correlation coefficient function in Eq.(4). The strain smoothness and tissue incompressibility terms penalize large strain gradients and deviations from incompressibility in velocity estimation. Further details regarding this estimator can be found in [5].
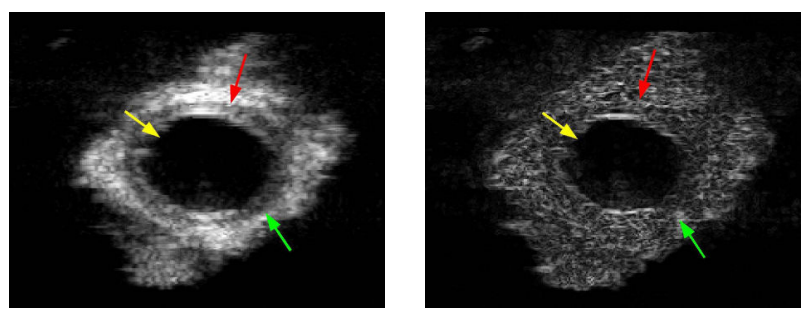

Figure 1. U/S image (left), gradient image (right).
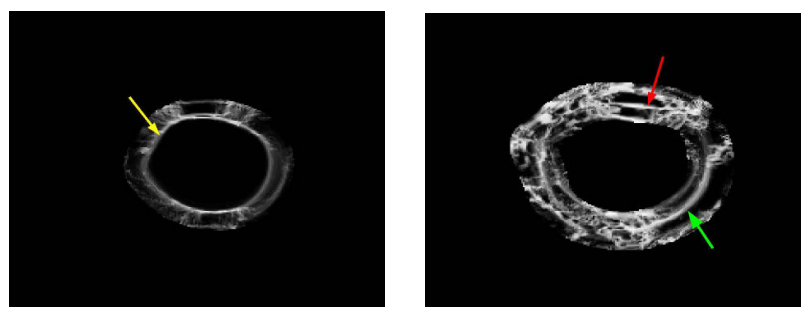

Figure 2. Enhanced edges; inner (left); outer (right).

The velocity estimates resulting from this process are used to compute a 2D deformation tensor $F$ at each point $(x, y)$ in the arterial wall:

$$
\mathbf{F}(x, y)=\left[\begin{array}{cc}
1+\frac{\partial u}{\partial x} & \frac{\partial u}{\partial y} \\
\frac{\partial v}{\partial x} & 1+\frac{\partial v}{\partial y}
\end{array}\right]
$$

The Green-Lagrange strain $\mathbf{E}$ at each wall location is then computed from $\mathbf{F}$, as follows:

$$
\mathbf{E}(x, y)=\frac{1}{2}\left\{\mathbf{F}^{T} \mathbf{F}-\mathbf{I}\right\}
$$

where $\mathbf{I}$ is the identity matrix. The eigenvalues of the strain tensor $\mathbf{E}$ are then calculated to obtain the principal strains at all locations within the arterial cross-section.

\section{Results and discussion}

Fig. 1 - 2 illustrate enhanced edge fields from our 3D directionally-sensitive, edge preserving filter. Parameters used for smoothing of the inner lumen boundary were $\alpha_{x}=10, \alpha_{y}=30, \alpha_{z}=50, \beta=0.03$, and $\rho=0.002$. For the outer boundary, $\alpha_{x}=20, \alpha_{y}=60$, and $\alpha_{z}=100$, $\beta=0.08$, and $\rho=0.003$, were used. Arrows highlight areas where the lumen boundaries are barely detectable in the gradient edge field, yet they can be detected in the enhanced edge fields.

After the 3D enhanced edge fields were obtained for the carotid volumes, a deformable surface was applied to each volume to extract the vessel boundaries. For the inner boundary the parameters used were: $a=0.0006$, $b=0.0006$. For the outer boundary, $a=0.01, b=0.02$.

IMT measurements were calculated from the 3D inner and outer boundaries that were extracted with the de- 


\begin{tabular}{|l|l|l|}
\hline Intra- & Mean Difference (mm) & $\begin{array}{c}95 \% \text { Conf. } \\
\text { interval }(\mathrm{mm})\end{array}$ \\
\hline \hline Manual & $-0.006(\sim 0.6 \%$ of IMT) & 0.277 \\
\hline Auto & $0.007(\sim 0.7 \%$ of IMT) & 0.234 \\
\hline Manual-Auto & -0.013 & 0.043 \\
\hline \multicolumn{3}{|l|}{} \\
\hline Inter- & Mean Difference (mm) & $\begin{array}{c}95 \% \text { Conf. } \\
\text { interval }(\mathrm{mm})\end{array}$ \\
\hline \hline Manual & $0.095(\sim 9.5 \%$ of IMT) & 0.368 \\
\hline Auto & $0.025(\sim 2.5 \%$ of IMT) & 0.288 \\
\hline Manual-Auto & 0.070 & 0.080 \\
\hline
\end{tabular}

Table 1. Intra- and inter-observer IMT reproducibility.
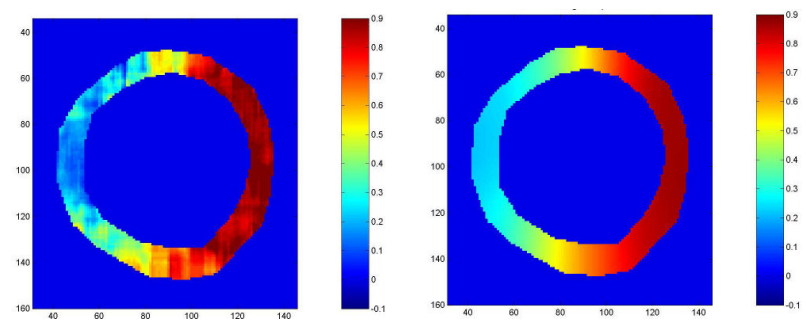

Figure 3. Conventional vs. robust $u$ velocities.

formable surface. These measurements were compared to those calculated using manual point selection of the inner and outer boundaries. Results from Bland-Altman analysis are summarized in Table 1 [6].

In all 3D scans analyzed, Bland-Altman plots show that for both intra- and inter- observer results, reproducibility for mean IMT measurements for each cross-section in the volume is better when using auto-tracing vs. manual tracing.

In Fig. 3, one can see that the velocity fields resulting from our robust method for velocity estimation qualitatively produce a much smoother velocity field than the conventional methods. Also, the regions where the velocity estimate is high agree with visual assessment of the arterial wall motion. It then follows that the corresponding maximum principle strain in the vessel wall for the robust technique is also smoother than the conventional method (Fig. 4). Lastly, strains of $\sim 1 \%$ are reasonable for the deformation seen in the image sequence used.

\section{Conclusions}

Our directionally-sensitive edge preserving filter applied to U/S images extracts more distinct edge vessels and thus more reproducible IMT measurements than conventional methods. Also, our methods for velocity and strain estimation are more robust to imaging noise than conventional methods.
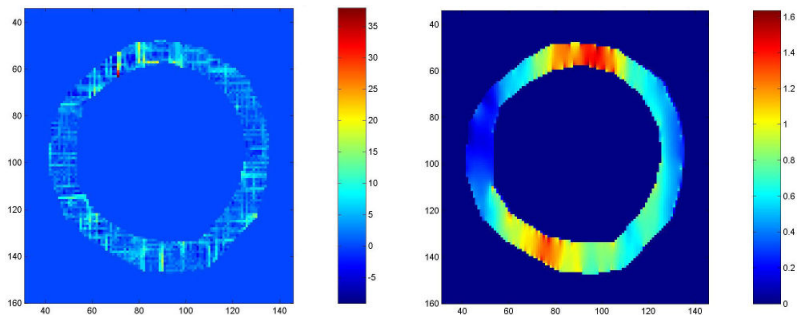

Figure 4. Conventional vs. robust strains.

\section{Acknowledgements}

Research funding was provided by the NIH under training grant T-32 EB2102, by the NSF grant GRF \# DGE0202745-10, by the Department of Defense through the Center for Integration of Medicine and Innovative Technology (CIMIT Vulnerable Plaque Program), and by the Boston Heart Foundation.

\section{References}

[1] Pignoli P, Tremoli E, Poli A, Oreste P, Paoletti R. Intimal plus medial thickness of the arterial wall: a direct measure with ultrasound imaging. Circulation 1986;74:1399-1406.

[2] Kaazempur-Mofrad M, Isasi A, Younis H, Chan R, Hinton D, Sukhova G, LaMuraglia G, Lee R, Kamm R. Characterization of the atherosclerotic carotid bifurcation using MRI, finite element modeling, and histology. Annals of Biomedical Engineering 2004;32(7):932-946.

[3] Shah J. A common framework for curve evolution, segmentation, and anisotropic diffusion. In Proceedings of CVPR 96. San Francisco: IEEE Computer Society Press, 1996; 136-142.

[4] Chan RC. Non-invasive ultrasound monitoring of regional carotid wall structure and deformation in atherosclerosis. Ph.D. thesis, Massachusetts Institute of Technology, 77 Massachusetts Ave., Cambridge MA, May 2001.

[5] Chan R, Chau AH, Karl W, Nadkarni S, Khalil A, Iftimia N, Shishkov M, Tearney G, Kaazempur-Mofrad M, Bouma B. OCT-based arterial elastography: robust estimation exploiting tissue biomechanics. Optics Express 2004;12(19):45584572.

[6] Bland JM, Altman DG. Statistical methods for assessing agreement between two methods of clinical measurement. Lancet 1986;1(8476):307-310.

Address for correspondence:

Raymond C. Chan, Ph.D.

Bartlett Hall, Rm 710, Boston, MA, 02114

rchan@ nmr.mgh.harvard.edu

${ }^{*}$ K. Dionisio and R. Chan are co-first-authors 